\title{
Management of hematologic toxicity in patients with advanced or metastatic gastric cancer treated with docetaxel, cisplatin and fluorouracil (DCF): Results of monocentric experience
}

\author{
Turano Salvatore ${ }^{1,2}$, Biamonte Rosalbino ${ }^{1}$, Conforti Serafino ${ }^{1}$, Mastroianni Candida Maria ${ }^{1}$, \\ Manfredi Caterina ${ }^{1}$, Palazzo Salvatore ${ }^{1}$ \\ ${ }^{1}$ U. O. C. Oncologia Medica S. O. "Mariano Santo" Azienda Ospedaliera Cosenza \\ ${ }^{2}$ Ospedale Mariano Santo UOC Oncologia Medica (dir S. Palazzo) c/da Muoio Piccolo 87100 Cosenza, Italy
}

Email address:

galactus76@yahoo.it (Turano S.)

\section{To cite this article:}

Turano Salvatore, Biamonte Rosalbino, Conforti Serafino, Mastroianni Candida Maria, Manfredi Caterina, Palazzo Salvatore. Management of Hematologic Toxicity in Patients with Advanced or Metastatic Gastric Cancer Treated with Docetaxel, Cisplatin and Fluorouracil (DCF): Results of Monocentric Experience. Clinical Medicine Research. Vol. 3, No. 6, 2014, pp. 166-170. doi: 10.11648/j.cmr.20140306.12

\begin{abstract}
Background: Gastric adenocarcinoma is the second most common cause of cancer death worldwide. There is no standard regimen of chemotherapy for metastatic disease, although the regimen of ECF is the most used regimen, with a median survival of 7-9 months. With new regimens of chemotherapy, such as DCF, the median survival has increased, despite a major toxicities and 1-2\% of toxic death.Patients And Methods: This is a monocentric experience. In three years (2009-2012) we have treated 36 chemo-naïve patients with histological diagnosis of locally advanced or metastatic gastric cancer with a DCF regimen. All patients were treated with a prophilactic Peg-filgrastim injection at 6th day of therapy. Results: A total of 168 cycles were administered (median 5 per patient, range 3-8). Major responses were observed in 10 patients, with 2 complete $(5,5 \%)$ and 8 partial remissions $(22,2 \%) ; 16$ additional pts showed disease stabilization $(44,4 \%)$ and 10 progressed $(27,9 \%)$. Median OS times were 12 months. Median TTP were 9,5 months. Toxicity was acceptable, worst per patient toxicities were neutropenia (grade 3-4 in 15\%), feverish neutropenia (11,1\%) diarrhoea (grade 2 in $25 \%$, grade 3 in $25 \%$, grade 4 in $18,8 \%$ ), asthenia (grade 2 in $8 \%$ ), neurotoxicity (grade 3 in $4 \%$ ), anhemia (grade 4 in 10\%), four pts received blood transfusion. Conclusion: Time to response and ORR favor DCF over other schedule's treatment according to literature. A trend towards increased myelosuppression and infectious complications was observed but the management of this and others side-effects is possible and not too difficult if pts is managed by an expert toxicities team. Infact we don't have no-one toxic death.
\end{abstract}

Keywords: Gastric Cancer, DCF, Hematologic Toxicity

\section{Introduction}

Gastric cancer is one of the most frequent malignancies in Europe since the fifth cancer incidence for both the male and in female. Over the past decades, the epidemiology of gastric cancer has been profoundly modified by a significant decrease in incidence and mortality. The incidence and mortality are widely different in different regions of the world. In Europe there are about 192,000 new cases per year with an incidence 1.6 times higher in males. The annual incidence rates standardized by age show a greater incidence in Eastern Europe $(34.1 / 100,000)$ and South $(19.5 / 100,000)$ than Western Europe $(7 / 100.000)$ and North $(6.1 / 100,000)^{1}$.
The precise number of cancer cases diagnosed each year in Italy is unknown because complete cancer registration has not yet been achieved in many regions.

Despite the presence of numerous epidemiological studies, the etiology of gastric cancer is not perfectly known. The gastric carcinogenesis is a multifactor process with progressive deterioration and involving environmental irritants, bacterial infections, changes in acid secretion, production of nitrite and $\mathrm{N}$ - nitrous compounds derived from food. The results of this cascade of events result in a gradual transition from normal epithelium to gastric cancer. There were many assumptions and interpretations have been relied on ethnicity, economic status, blood group and type of 
nutrition, also were deemed responsible for pathological conditions such as achlorhydria defined predisposing or ipocloridria, pernicious anaemia, gastric polyposis, the atrophic gastritis, intestinal metaplasia gastric and chronic ulcer. A prognostic factor of great importance is represented by a previous Billroth II gastric resection type, for a possible mucolytic action of bile reflux and increased production of nitrosamines as a result of the presence of intestinal bacterial flora and the changes in $\mathrm{pH}^{1}$

In Italy gastric cancer is the 5 th in order of cancer incidence with almost 17000th new cases per year (5th in men after lung, colorectal, bladder and prostate and 3rd in women after breast and colorectal). Although in Italy there is any decline in mortality, this seems to reflect the parallel decline in the incidence, well documented, even in our country, from the mid 70s.

Even today, in the Western world, over $60 \%$ of patients come to surgery in Stage III or IV ${ }^{\circ}$ (pT2 N2, T3 N1, T4 N0, T3 N2, T4 N1, T4 N2, M1), and also for patients undergoing curative surgery the 5-year survival does not exceed $15-30 \%$ and loco-regional recurrence (38-67\%) and distance (25-52\%) are divided almost equally responsible for this high mortality ${ }^{25}$. Clearly, therefore demonstrates the importance of a therapeutic approach focuses on advanced or recurrent forms, in fact over the past 2 decades have seen, all over the world, numerous efforts by surgeons, radiation oncologists and physicians to achieve better results. Particularly in the field of chemotherapy for advanced or recurrent forms there has been the definitive proof that the modern chemotherapy schedules have an advantage over best supportive care ca, $^{2,4,5}$, and, almost certainly on older single agent approaches. However, no protocol, among those currently available, can be considered the 'gold standard', as many are equivalent in terms of ORR is PFS or $\mathrm{OS}^{6,9}$ because the results obtained so far in terms of OS (8-12 months ) are still widely considered inadequate.

The possibility that, as yet, practically, before new therapies are made to finalize (eg angiogenesis inhibitors, vaccinations etc ...), go through the optimization of the use of existing drugs (new modalities and timing of administration) and their associations (new combination therapies).

More recently highlighted the effectiveness of combination chemotherapy such as docetaxel and cisplatin $(\mathrm{TC})^{13}$, Docetaxel, cisplatin and 5-fluorouracil (TCF) ${ }^{14}$.

In a recent randomized phase II combination DCF (docetaxel $85 \mathrm{mg} / \mathrm{m} 2$ day 1 , cisplatin $75 \mathrm{mg} / \mathrm{m} 2$ day $1,5-$ Fluorouracil $300 \mathrm{mg} / \mathrm{m} 2 /$ die in IC 2 weeks every 3 weeks) achieved better results (which are still supported by a randomized phase III) compared to TC and ECF, reporting an overall response rate respectively of $55 \%, 42 \%$ and $46 \%$ and a median time to progression of $7.3,4.3$ and 5.0 months ${ }^{15}$

Another randomized phase III study comparing the pattern DCF (docetaxel $75 \mathrm{mg} / \mathrm{m} 2$ days 1 , cisplatin $75 \mathrm{mg} / \mathrm{m} 2$ days 1 , 5 -Fluorouracil $750 \mathrm{mg} / \mathrm{m} 2 /$ die in IC on days $1-5$, every 3 weeks) combined with CF (cisplatin $100 \mathrm{mg} / \mathrm{m} 2$ days 1 and 5 -Fluorouracil in $1000 \mathrm{mg} / \mathrm{m} 2 /$ die CI on days $1-5$ every 4 weeks) showed a benefit of DCF regarding: median time to progression (5.6 vs 3.7 months), survival at 2 years (18\% vs $9 \%)$ and overall response rate $(37 \%$ vs $25 \%)$ with an acceptable toxicity profile for the association of three $\operatorname{drugs}^{8,16}$.

In view of these findings is important to the management of toxicities' haematological (neutropenia and febrile neutropenia) and in the schedule are higher than DCF and CF, especially in elderly patients and those with comorbidities or performance status deteriorated ${ }^{11}$.

The introduction of growth factors in the early 90 'has had and continues to have a profound impact on oncology practice. The numerous studies of these cytokines have enabled us to gather a lot of information about both their biological effects on both the indirect effects resulting from the reduction of neutropenia, anemia or thrombocytopenia ${ }^{17,18}$.

Several $\mathrm{mAbs}$ and kinase inhibitors, especially those targeting EGFR and VEGF/VEGFR, have already demonstrated promising activity in gastric cancer. Trastuzumab was the first targeted agent to be approved for the treatment of advanced gastric cancer in 2010. The Phase III ToGA trial reported an increase in overall survival for patients with human EGF receptor (HER)2-positive gastric cancer treated with chemotherapy and trastuzumab compared to chemotherapy alone $\mathrm{e}^{33,34}$. The failure of the AVAGAST trial $^{35}$ was a setback for anti-angiogenic therapy for this disease. Ramucirumab ${ }^{36}$ is a monoclonal antibody that binds to VEGF-R2 and prevents its activation. The recent REGARD trial was a randomized phase III trial of ramucirumab vs. placebo for patients with advanced, pretreated gastric cancer that met its primary endpoint of increased overall survival

With specific reference to growth factors granulocyte (G$\mathrm{CSF}$ ) and granulocyte-macrophage (GM-CSF), the stock of knowledge continues to expand today: in terms of clinical and pathophysiological point of view, making this class of drugs among most 'versatile oncohaematology ${ }^{19,20}$.

The growth factors granulocyte intervene directly on the natural history and prognosis of the disease and then use the G-CSF mean, in light of the results of 15 years of large clinical trials and several meta-analysis, allowing the patient a better prognosis and better quality of life $\mathrm{e}^{21,22}$.

We wanted to assess the safety. The primary objective of this study is to assess the impact of pegfigrastim on neutropenia induced by combination chemotherapy with paclitaxel, 5-fluorouracil (5-FU) and cisplatin for treating advanced gastric cancer. The secondary objective of the current study was to examine the other side effects.

\section{Patients and Methods}

From January 2009 to September 2012 were enrolled at our center, OU Of Medical Oncology, Azienda Ospedaliera di Cosenza, 36 patients with histological diagnosis of locally advanced or metastatic gastric cancer. Inclusion criteria were represented by: gastric or gastroesophageal junction 
adenocarcinoma histologically documented, measurable metastatic and / or assessed according to WHO criteria, when present, or at least 1 local recurrence with lymph node measured, Age $>18$ years, PS sec. ECOG 0-2, life expectancy $>3$ months, adequate hematologic function $(\mathrm{Hb} \geq$ $10 \mathrm{~g} / \mathrm{dl}$, neutrophils $\geq 1500$, platelets $\geq 10.000 \mathrm{MMC}$ ), creatinine clearance $\geq 30 \mathrm{ml} / \mathrm{min}$., Adequate liver function: bilirubin $\leq 1.25 \times \mathrm{VNM}$ (or $\leq 1.5 \mathrm{x} \mathrm{VNM}$ in the presence of liver metastases), SGOT and SGPT $\leq 1.5 \times$ VNM (or $\leq 2.5$ $\mathrm{xVNM}$ in the presence of liver metastases), normal cardiac function: ejection fraction $\geq 50 \%$ assessed echocardiography, patient compliance and geographic conditions that allow attachment to the follow-up.

Main exclusion criteria were as follows: prior palliative chemotherapy or prior treatment with taxanes; symptomatic peripheral neuropathy; known brain or leptomeningeal metastases; liver impairment with ALT and/or AST more than $1.5 x$ the upper limit of normal associated with alkaline phosphatase more than $2.5 \mathrm{x}$ the upper limit of normal; and other serious medical condition that could impair participation in the study. Written informed consent was obtained from all patients.

Before starting treatment, patients underwent complete medical examination, evaluation of performance status (ECOG), measurement of metastatic lesions with ultrasound or CT scans where possible. All patients were treated with a regimen: docetaxel $75 \mathrm{mg} / \mathrm{m} 2$ in 1 hour infusion, CDDP 75 $\mathrm{mg} / \mathrm{m}^{2} \mathrm{IV} 1$ to 3 hours of infusion $1 \mathrm{~g}, 5-\mathrm{FU} 750 \mathrm{mg} /$ $\mathrm{m} 2 /$ die continuous infusion for 5 days (DCF) every three weeks until disease progression or onset of severe toxicity. All patients also received prophylactic treatment with PEGfilgrastim $6 \mathrm{mg}$ on the sixth day of every cicles of therapy. The patients received an average relative dose intensity of $>/=75 \%$. Less than $15 \%$ of patients experienced a dose delay, and $<10 \%$ of patients received a chemotherapy reduction Responses were assessed at the end of each cycle according to WHO criteria and toxicity every week according to NCI CTC. Patients who have completed at least one cycle of chemotherapy were considered evaluable for toxicity.

The follow-up was carried out over a year after the last cycle of chemotherapy, and was carried out with medical consultation and blood chemistry every 3 months, CT abdomen and chest X-ray every 6 months.

\section{Results}

Were administered a total of 168 treatment cycles with an average of 5 cycles per patient (range 3-8). Responses to therapy: complete response (CR) $2(5.5 \%)$ partial response (PR) 8 (22.2\%), stabilization disease (SD) 16 (44.4\%), progression disease (PD) $10(27.9 \%)$. The median overall survival was 12 months while the median time to progression was of 9.5 months. The toxicity 'has been accepted. In particular, regarding hematological toxicity, we observed: grade 3-4 neutropenia in (15\%), febrile neutropenia $(11.1 \%)$, grade 4 anemia (10\%). Toxicities' non-haematological were: diarrhea grade $2(25 \%)$ grade $3(25 \%)$ grade $4(18.8 \%)$, grade 2 asthenia (8\%) neurotoxicity 'grade 3-4 (4\%). Only 4 patients received blood transfusion.

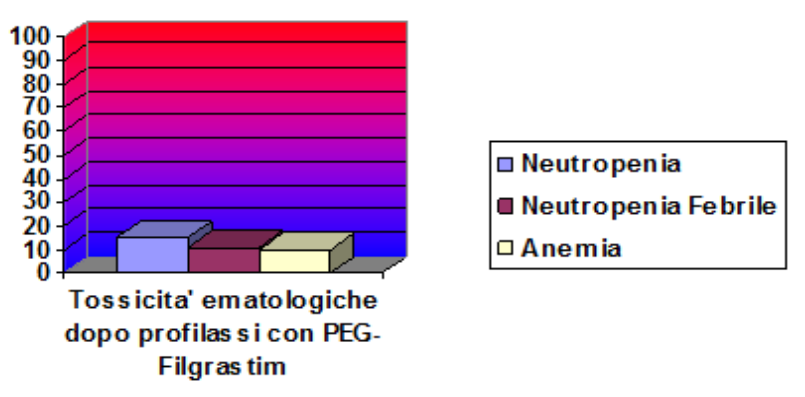

Figure 1. Hematologic Toxicity's after prophylaxis with PEG-filgrastim.

\section{Discussion}

The V325 trial is the largest randomized, controlled trial that formally and systematically assessed QoL in patients with advanced gastric cancer or gastroesophageal junction adenocarcinoma ${ }^{23}$.

In summary, the V325 randomized phase III trial results in a significantly better preservation of QoL for patients treated with DCF than patients treated with CF. In this study, better preservation of QoL occurred as a result of a significantly higher level of efficacy imparted by the addition of docetaxel to CF compared with $\mathrm{CF}$ alone despite a higher incidence of some toxicities as a result of the addition of docetaxel to CF. Clearly, the quantity and quality of life are important outcomes and, ideally, treatment should enhance both. The research on QoL provides a qualitative and quantitative background to integrate these elements into the decisionmaking process. The QoL results of the V325 study consistently favored DCF over CF, supporting the benefit of DCF in patients with advanced gastric or gastroesophageal junction adenocarcinoma ${ }^{24}$.

The hematologic toxicity represented and represent a daily problem in clinical practice for the specialist oncologist both in the elderly patient and young patient management.

The introduction of granulocyte growth factors as part of the treatment plan has allowed us to intervene directly on the prognosis and natural history of disease.

A number of initiatives are under way to begin to measure and improve the quality of cancer care, including the American Society of Clinical Oncology's (ASCO's) Quality Oncology Practice Initiative, ${ }^{26}$ the National Initiative for Cancer Care Quality (NICCQ), ${ }^{27}$ and the Oncology Demonstration Project sponsored by the Centers for Medicare and Medicaid Services. ${ }^{28}$ In addition to improving the quality of care associated with the diagnosis, staging, and cancer-related therapy, an equally important priority is to ensure that patients receive appropriate supportive care to manage both disease symptoms and treatment-related toxicity. $^{29,30,31}$.

In our study, we want to evaluate the impact of pegfigrastim on the neutropenia chemotherapy-related. Our second goal is to highlight how the toxicities due to DCF 
thrice-weekly schedule for the treatment of gastric cancer can be reduced and controlled if the patient is followed by a team experienced in the toxicity management.

36 patients with locally advanced or metastatic gastric cancer were enrolled and treated with a schedule Docetaxel $75 \mathrm{mg} / \mathrm{m} 2$, CDDP $75 \mathrm{mg} / \mathrm{m} 2$, 5FU $750 \mathrm{mg} / \mathrm{m} 2$ every three weeks.

The responses, when compared with the data reported for other schedules as reported in the literature, were in favor of DCF in terms of both TTR and ORR, both for overall survival and time to progression.

The prophylactic treatment with PEG-filgrastim administered on the sixth day of therapy in all patients, has allowed a major reduction and better management of hematological toxicities when compared with data of TAX 325 study $^{16}$.In this study there was no requirement to use growth factors granulocyte toxicity and there was hematological neutropenia grade 3-4 (82\%), febrile neutropenia (29\%), grade 3-4 anemia (18\%).

In our experience, we have associated with chemotherapy treatment a granulocyte growth factor using PEG-filgrastim on the sixth day of therapy. This enabled us to significantly reduce the hematologic toxicity of allowing the patient a better prognosis and a better quality of life (QoL).

Therefore, more than fifteen years after the introduction of growth factors in oncology, there is no doubt that they are an essential for achieving various objectives garrison including QoL.The limit of our study consists in amance of a control group.

\section{Conclusions}

It's important to stress that the results were obtained in a group of patients more realistic (over seventy patients with diseases associated) and less than that explored in selected studies, and for that reason has been the subject of criticism in the months following the publication .

These findings underscore the need for observational research to evaluate the effectiveness of therapy in heterogeneous patient populations treated in our oncology unit. While all new U.S. pharmaceutical therapies undergo rigorous evaluation to demonstrate their safety and efficacy during the FDA approval process, variations in actual use and the diversity of patient populations may have a small but measurable impact on the effectiveness of medications. ${ }^{32}$

It is also important to stress that in our study there were no deaths from toxic effects compared with an incidence of 3-7\% in the literature, despite the characteristics of the patients expressed above. This is primarily due to a good and timely management of expectations and toxicity prevention performed with peg-filgrastim.

We conclude that the use of prophylactic growth factor support was associated with a high proportion of chemotherapy cycles that were delivered on schedule and at full dose.

Our monocentric experience confirms that the DCF scheme in locally advanced or metastatic gastric carcinoma is a scheme with an acceptable toxicity even in not highly selected patients. Of course, the results of our study will be confirmed in randomized phase III multicenter studies.

\section{References}

[1] La Bianca R., Beretta G., Bozzetti F., Milesi L., Mosconi S. Quadri A. . Neoplasie dell'apparato digerente da G. Bonadonna , G.Robustelli Della Cruna , P. Valagussa , MEDICINA ONCOLOGICA VIII ed. Masson Milano 1025 1026.2007

[2] Murad AM., Santiago FF., Petroianu A., et al. "Modified therapy with 5-fluorouracil, doxorubicin and methotrexate in advanced gastric cancer". Cancer;72:37.1993

[3] Glimelius B., Hotfmann K., Haglund U., et al. "Initial or delayed chemotherapy with best supportive care in advanced gastric cancer”. Ann Oncol;5:189. 1994

[4] Pyrhönen S., Kuitumen T., Kouri M. "A randomized phase III trial comparing fluorouracil, epidoxorubicin and methotrexate (FEMTX) with best supportive care in non resectable gastric cancer”. Ann Oncol;3(Suppl 5):12. 1992

[5] Scheithauer W., Kornck G., Zeh B., et al. "Palliative chemotherapy versus supportive care in patients with metastatic gastric cancer: a randomized trial". Second International Conference on Biology, Prevention and Treatment og GI Malignancy, Köln, Germany,:68. 1995

[6] Schipper DL, Wagener DJT et al: Chemotherapy of gastric cancer. Anticancer Drugs, 7: 137-149, 1996.

[7] Cascinu S: La gestione del paziente con carcinoma gastrico. Tumori vol 1, n 1: S3-S10, 2002.

[8] Wils JA, Klein HO, Wagener $\mathrm{H}$ et al: Sequential high-dose methotrexate and fluorouracil combined with doxorubicin-a step ahead in the treatment of advanced gastric cancer: a trial of the European Organization for Research and Treatment of Cancer Gastrointestinal Tract Cooperative Group. J. Clin. Oncol. 9: 827-831, 1991.

[9] Cocconi G, Bella M, Zironi S et al: Fluorouracil, doxorubicin, and mitomycin combination versus PELF chemotherapy in advanced gastric cancer: a prospective randomized trial of the Italian Oncology Group for Clinical Research. J. Clin. Oncol. 12: 2687-2693, 1994.

[10] Cocconi G, Carlini P, Gamboni A et al: PELF is More Active than FAMTX in Metastatic Gastric Carcinoma (MGC) Proc. Am. Soc. Clin. Oncol. Abst. 501. 2001

[11] Waters JS, Norman A, Cunningham D et al: Long-term survival after epirubicin, cisplatin and fluorouracil for gastric cancer: results of a randomized trial. Br. J. Cancer 80: 269-72, 1999.

[12] Vanhofer U, Rougier P, Wilke $\mathrm{H}$ et al: Final results of a randomized phase III trial of sequential high-dose methotrexate, fluorouracil and doxorubicin versus etoposide, leucovorin and fluorouracil versus infusional fluorouracil and cisplatin in advanced gastric cancer: A trial of the European Organization for Research and Treatment of Cancer Gastrointestinal Tract Cancer Cooperative Group. J. Clin. Oncol. 18: 2648-2657, 2000.

[13] Roth AD et al. Ann Oncol; 11:301;). 2000

[14] MoiseyenkoV et al. ASCO, Abstr 587. 2002 
[15] Roth AD et al. ASCO, Abstr 4020. 2004

[16] Van Cutsem E, Moiseyenko VM, Tjulandin S et al. Phase III study of docetaxel and cisplatin plus fluorouracil compared with cisplatin and fluorouracil as firstline therapy for advanced gastric cancer: A report of the V325 study group. J Clin Oncol; 24: 4991-4997. 2006

[17] Bhana N. Granulocyte colony-stimulating factors in the management of chemotherapy-induced neutropenia : evidence based review. Curr Opin Oncol ;19:328-335. 2007

[18] Pro B, Fayad L, McLaughlin P,et al. Pegfilgrastim administered in a single fixed dose is effective in inducing neutrophil count recovery after paclitaxel and topotecan chemotherapy in patients with relapsed aggressive nonHodgkin's lymphoma. Leuk Lymphoma;47:481-485. 2006

[19] Lyman GH . Pegfilgrastim: a granulocyte colony-stimulating factor with sustained duration of action . Expert Opin Biol Ther;5:1635-1646. 2005

[20] Rader M. Granulocyte colony-stimulating factor use in patients with chemotherapy-induced neutropenia : clinical and economic benefits . Oncology ( Williston Park ); 20(5 Suppl 4) 16-21. 2006

[21] Andre N , Kababri ME, Bertrand P, et al. Safety and efficacy of pegfilgrastim in children with cancer receiving myelosuppressive chemotherapy . Pharmacotherapy; 23(8 Pt 2) : 15S-19S. 2003

[22] Crawford J , Dale DC , Lyman GH. Chemotherapy-induced neutropenia: risks, consequences, and new directions for its management. Cancer; 100:228-237. 2004

[23] Ajani JA, Moiseyenko VM, Tjulandin S, et al. Quality of Life With Docetaxel Plus Cisplatin and Fluorouracil Compared With Cisplatin and Fluorouracil From a Phase III Trial for Advanced Gastric or Gastroesophageal Adenocarcinoma: The V-325 Study Group. J. Clin. Oncol. 22: 3210-3216, 2007.

[24] Ajani JA, Moiseyenko VM, Tjulandin S, et al: Clinical benefit with docetaxel plus fluorouracil and cisplatin compared with cisplatin and fluorouracil in a phase III trial of advanced gastric or gastroesophageal cancer adenocarcinoma: The V-325 Study Group. J Clin Oncol 25:3205-3209, 2007

[25] Dicken BJ, Bigam DL, Cass C, Mackey JR, Joy AA, Hamilton SM. Gastric adenocarcinoma. Review and considerations for future directions. Ann Surg (2005) 241:2739.
[26] Neuss MN, Desch CE, McNiff KK, et al. A process for measuring the quality of cancer care: the Quality Oncology Practice Initiative. J Clin Oncol. 2005;23:6233-39.

[27] Schneider EC, Epstein AM, Malin JL, et al. Developing a system to assess the quality of cancer care: ASCO's national initiative on cancer care quality. J Clin Oncol. 2004;22:298591.

[28] Twombly R. Medicare demonstration projects acknowledge evidence-based medicine in cancer care. J Natl Cancer Inst. 2005;97:6-7.

[29] Lipscomb J, Donaldson MS. Outcomes research at the National Cancer Institute: measuring, understanding, and improving the outcomes of cancer care. Clin Ther.;25:699-712. 2003

[30] Malin JL. Bridging the divide: integrating cancer-directed therapy and palliative care. J Clin Oncol.;22:3438-40. 2004

[31] Patrick D, Ferketich S, Frame P, et al. National Institutes of Health State-ofthe-Science Panel. National Institutes of Health State-of-the-Science conference. July 15-17. 2002

[32] Agency for Healthcare Research and Quality. Outcomes of Pharmaceutical Therapy Program (OPT) update. AHRQ Publication No. 04-R205, Agency for Healthcare Research and Quality, Rockville, MD: Agency for Healthcare Research and Quality. Available at: http://www.ahrq.gov/clinic/pharmtherapy/ optupdat.htm. Accessed June 2004.

[33] De Vita F, Giuliani F, Silvestris N, Rossetti S, Pizzolorusso A, Santabarbara G, Galizia G, Colucci G, Ciardiello F, Orditura M. Current status oftargeted therapies in advanced gastric cancer Expert Opin Ther Targets. 2012 Apr;16 Suppl 2:S2934. doi: 10.1517/14728222.2011.652616. Epub Mar 23. 2012

[34] Giuffrè G, Ieni A, Barresi V, et al. HER2 status in unusual histological variants of gastric adenocarcinomas. J Clin Pathol.;65:237-241. 2012

[35] Bang YJ, Van Cutsem E, Feyereislova A, Chung HC, Shen L, Sawaki A, et al. Trastuzumab in combination with chemotherapy versus chemotherapy alone for treatment of HER2-positive advanced gastric or gastro-oesophageal junction cancer (ToGA): a phase 3, open-label, randomised controlled trial. Lancet.;376:687-697. doi: 10.1016/S01406736(10)61121-X. 2010

[36] Javle M, Smyth EC, Chau I. Ramucirumab: Successfully Targeting Angiogenesis in Gastric Cancer. Clin Cancer Res. 2014 Oct 3. pii: clincanres.1071. [Epub ahead of print] 2014 